\title{
Evaluation of Performance Enhancement of Optical Multi-Level Modulation Based on Direct Modulation of Optically Injection-Locked Semiconductor Lasers
}

\author{
Hyo-Sang Jeong ${ }^{1}{ }^{\mathbb{C}}$, Jun-Hyung Cho ${ }^{2}$ and Hyuk-Kee Sung ${ }^{1, *}$ \\ 1 School of Electronic and Electrical Engineering, Hongik University, Seoul 04066, Korea; \\ B515186@mail.hongik.ac.kr \\ 2 Research Institute of Science and Technology, Hongik University, Seoul 04066, Korea; \\ kadokal@mail.hongik.ac.kr \\ * Correspondence: hksung@hongik.ac.kr
}

check for updates

Citation: Jeong, H.-S.; Cho, J.-H.;

Sung, H.-K. Evaluation of

Performance Enhancement of Optical Multi-Level Modulation Based on Direct Modulation of Optically Injection-Locked Semiconductor

Lasers. Photonics 2021, 8, 130.

https://doi.org/10.3390/photonics 8040130

Received: 31 March 2021

Accepted: 17 April 2021

Published: 19 April 2021

Publisher's Note: MDPI stays neutral with regard to jurisdictional claims in published maps and institutional affiliations.

Copyright: (c) 2021 by the authors. Licensee MDPI, Basel, Switzerland. This article is an open access article distributed under the terms and conditions of the Creative Commons Attribution (CC BY) license (https:/ / creativecommons.org/licenses/by/ $4.0 /)$
Abstract: The performance of optical M-level (multi-level) amplitude shift keying (ASK) modulation is improved by directly using modulated optically injection-locked (OIL) semiconductor lasers. The direct modulation performance of free-running and OIL semiconductor lasers is evaluated and compared theoretically based on coupled-rate equation. We have found that OIL semiconductor lasers can significantly improve the modulation performance in terms of the signal eye opening and Q-factor. Additionally, we found that the Q-factor increases even more in the negative frequency detuning range due to its dependence on the locking parameters.

Keywords: optically injection-locked; multi-level amplitude shift keying; direct modulation; semiconductor lasers

\section{Introduction}

The generation and transmission of high-capacity optical modulation signals have become key factors in recent photonic applications, such as high-speed optical communications, data centers, supercomputers, and photonic integrated circuits [1-4]. Accordingly, many studies have been conducted to support the demand for higher transmission speeds in optical communication. Optical modulation signals with high-speed, small-form factor, high output, and high efficiency should be accomplished for various next-generation optical systems to be utilized in real field applications.

Among the various technologies that enable high-speed optical data generation and signal processing, amplitude modulation (AM) is a widely known technology owing to its simplicity when compared to the other modulation methods such as frequency modulation (FM) or phase modulation (PM). FM and PM require a wider frequency bandwidth, and the electrical circuit of the transceivers is much more complex than that of AM. The AM method is realized either by a direction modulation of a laser or by external modulation of a laser using piezoelectric transducers, electro-optic modulators, or acousto-optic modulators [5-7]. However, external modulators have several limitations that hinder the performance of optical applications. They include a large form factor, high power consumption, and difficulty in integration with other optical devices [8]. Despite improved integration, challenges such as existence of parasitic modes and high insertion loss exist.

Optically injection-locked (OIL) semiconductor lasers have garnered considerable interest, as they exhibit various advantages over free-running lasers. In OIL semiconductor lasers, when light from a master laser (ML) is injected into a slave laser (SL), the output of the SL can be controlled to achieve the injection-locked state by the appropriate control of the injection locking parameters. In an OIL laser, the resonance frequency and modulation bandwidth of the SL can be improved [9-13]. It also exhibits improved laser performance such as reduction of mode partition noise [14], chirp [15-17], and nonlinear distortions [18,19]. Therefore, OIL 
systems are potential candidates for generating high-capacity signals for various photonic applications such as data centers, high-speed photonics integrated circuits, optical signal processing, and light detection and ranging.

High-speed direct modulation of a laser (DML) have been reported theoretically and experimentally in various optical communication applications [20-22]. Direct modulation of the OIL semiconductor laser can overcome the limitations of DML which includes the reduction of frequency chirp and harmful transient effects such as over and undershoots. The OIL laser ensures higher modulation speed owing to the enhanced resonant frequency. The enhanced modulation performances can be distributed to the array of slave lasers [23,24]. Coherent optical communications and applications can be also achieved by the OIL laser [15].

In this paper, we evaluate the performance of optical M-level modulation based on a directly modulated OIL semiconductor laser. We simulated the eye opening and the Q-factor of the optical M-level signal based on the coupled-rate equation. By observing the modulation signal from 2 to 32-level modulation with the symbol rate from 1 to $50 \mathrm{Gbaud}$, we concluded that performance of M-level modulation signal of the OIL semiconductor laser improved. Additionally, we evaluated the dependence of Q-factor on the locking parameters. We found that the Q-factor increases when the injection-locking parameters are adjusted in the region of negative detuning frequency and high injection ratio.

\section{Principle and Theoretical Model}

Figure 1 shows the schematic of the generation of the M-level ASK modulated optical signals based on OIL semiconductor lasers. The output of the ML is injected into the SL to achieve the injection-locked output of the SL. The amplitude and phase of the injectionlocked SL are controlled by the two injection-locking parameters: the detuning frequency and injection ratio. The detuning frequency is defined by the following Equation (1):

$$
\Delta \omega_{i n j} \equiv \omega_{\mathrm{ML}}-\omega_{f r, \mathrm{SL}}
$$

where $\omega_{\mathrm{ML}}$ and $\omega_{f r, S \mathrm{~L}}$ are the angular frequencies of ML and SL, respectively. The injection ratio is defined as the following Equation (2):

$$
R_{i n j} \equiv \frac{S_{\mathrm{ML}}}{S_{f r, \mathrm{SL}}}
$$

where $S_{\mathrm{ML}}$ is the number of photons of the ML and $S_{f r, \mathrm{SL}}$ is the number of photons in SL in the free-running state.

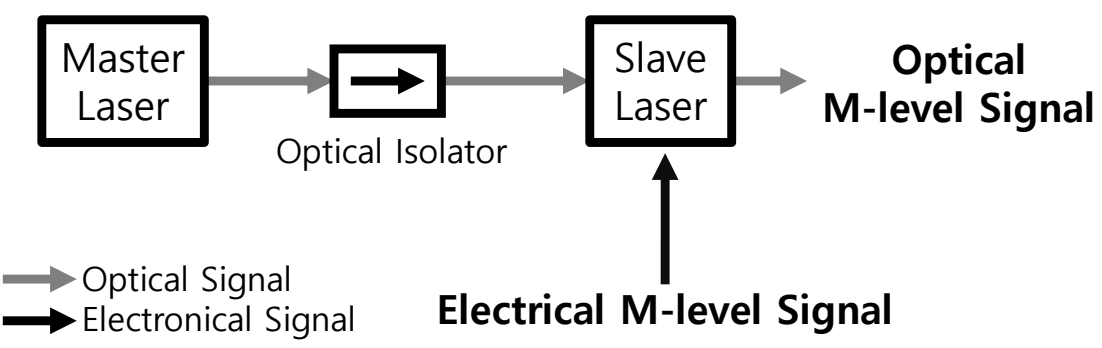

Figure 1. Schematic for the generation of the multi-level (M-level) amplitude shift keying (ASK) optical signals based on the optically injection-locked (OIL) semiconductor lasers.

The injection-locked SL is directly modulated by the electrical M-level signal to achieve an optical M-level signal. Several researchers have experimentally and theoretically investigated the modulation performance of semiconductor lasers based on rate equation analysis [25-27]. We evaluated the theoretical performance of the M-level signals using the coupled rate equation that describes the modulation process of OIL semiconductor lasers. For the evaluation, the exact relationship between the injection-locking parameters and the extent of AM that produces the desired M-level optical signals must be established first. 
Hence, we performed an analysis based on the rate equation, which includes the steady state and frequency response of the OIL semiconductor lasers. The rate equation for the OIL semiconductor lasers is defined as

$$
\begin{gathered}
\frac{d S(t)}{d t}=\left\{g\left[N(t)-N_{t r}\right]-\gamma_{p}\right\} S(t)+2 \kappa \sqrt{S_{\mathrm{ML}} S(t)} \cos \left[\phi(t)-\phi_{\mathrm{ML}}\right] \\
\frac{d \phi(t)}{d t}=\frac{\alpha}{2}\left\{g\left[N(t)-N_{t r}\right]-\gamma_{p}\right\}-\kappa \sqrt{\frac{S_{\mathrm{ML}}}{S(t)}} \sin \left[\phi(t)-\phi_{\mathrm{ML}}\right]-\Delta \omega_{i n j}, \\
\frac{d N(t)}{d t}=J(t)-\gamma_{n} N(t)-g\left[N(t)-N_{t r}\right] S(t),
\end{gathered}
$$

Equations (3)-(5) show the rate of change of photons and the number of free electron carriers in SL $[23,25]$. The time-dependent functions of $S(t), \phi(t)$, and $N(t)$ are the photon numbers, optical phase, and carrier numbers of the injection-locked SL, respectively. The time-dependent functions of $J(t)$ is the bias current. $S_{\mathrm{ML}}$ is the photon numbers of the ML. The physical parameters are defined as follows: $g$ is the linear gain, $N_{t r}$ is the transparency carrier number of free running SL, $\gamma_{p}$ is the photon decay rate, $\gamma_{n}$ is the carrier decay rate, $\kappa$ is the field coupling ratio between the ML and SL, and $\alpha$ is the linewidth enhancement factor of the laser. The values used for the calculation are listed in Table 1.

Table 1. Values used in calculations.

\begin{tabular}{cccc}
\hline Symbol & Quantity & Value & Unit \\
\hline$\lambda_{0}$ & Wavelength & 1550 & $\mathrm{~nm}$ \\
$g$ & Net stimulated gain & $4.7 \times 10^{4}$ & $1 / \mathrm{s}$ \\
$N_{t r}$ & Transparency carrier number & $9.36 \times 10^{6}$ & No unit \\
$J_{t h}$ & Threshold current & $2 \times 10^{16}$ & $1 / \mathrm{s}$ \\
$J_{b i a s}$ & Bias current & $5 \times J_{t h}$ & $1 / \mathrm{s}$ \\
$\gamma_{p}$ & Photon decay rate & $5 \times 10^{11}$ & $1 / \mathrm{s}$ \\
$\gamma_{n}$ & Carrier decay rate & $1 \times 10^{9}$ & $1 / \mathrm{s}$ \\
$\alpha$ & Linewidth enhancement factor & 5 & $\mathrm{No} \mathrm{unit}$ \\
$R_{i n j}$ & Coupling ratio & 225 & $1 / \mathrm{s}$ \\
$\Delta \omega_{i n j}$ & Injection power ratio & 10 & $\mathrm{~dB}$ \\
\end{tabular}

\section{Simulation and Result}

The small-signal modulation performance improvement and the potential for the complex signal generation of the OIL laser has been reported and found in $[24,25]$. In our simulation condition $\left(J=5 \times J_{t h}\right)$, we found that the frequency modulation response (not shown in this study) significantly enhanced the 3-dB modulation bandwidth from $17 \mathrm{GHz}$ for the free-running laser to $42 \mathrm{GHz}$ for the OIL laser. Here, we focus on the evaluation of the performance of the novel ultra-high-capacity optical signal generation. It is based on OIL semiconductor lasers directly modulated by M-level ASK signals.

Figure 2 shows the eye patterns of the semiconductor lasers modulated by M-level ASK signals under free-running conditions. For the simulation conducted in the present study, we biased the laser with a DC current of $5 \times J_{\text {th }}$ and modulated it with M-level signals equally divided by the value of $\mathrm{M}$ within the current range between the lowest level of $3 \times J_{t h}$ and the highest level of $7 \times J_{t h}$. It shows the output photon numbers for various M-levels and symbol rate. Figure $2 \mathrm{a}-\mathrm{c}$ show the eye patterns of 2-, 4-, and 8-level ASK modulated signals, respectively, at 1 -Gbaud data. Figure $2 d-f$ show the eye patterns of the 2-, 4-, and 8-level ASK modulated signals, respectively at a 5-Gbaud data. The higher symbol-rate and the increased number of M-levels significantly degrade the eye-opening performance, which hindered the ultra-high-speed optical signal generation and transmission. 


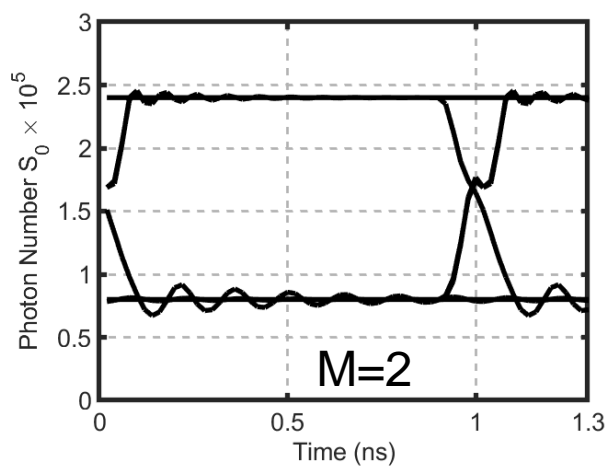

(a)

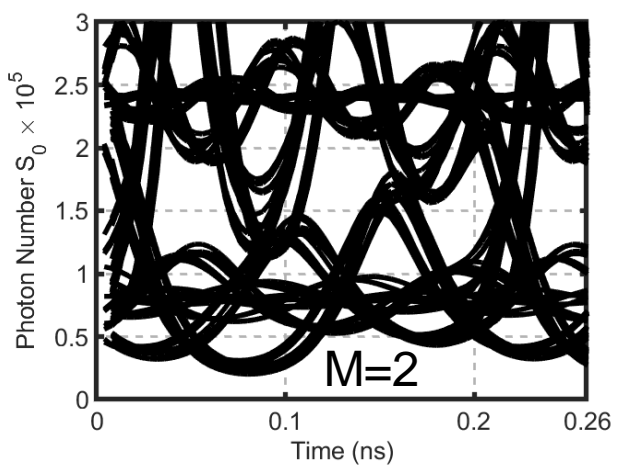

(d)

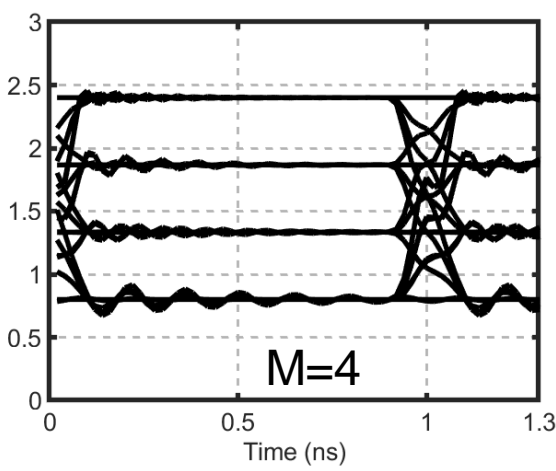

(b)

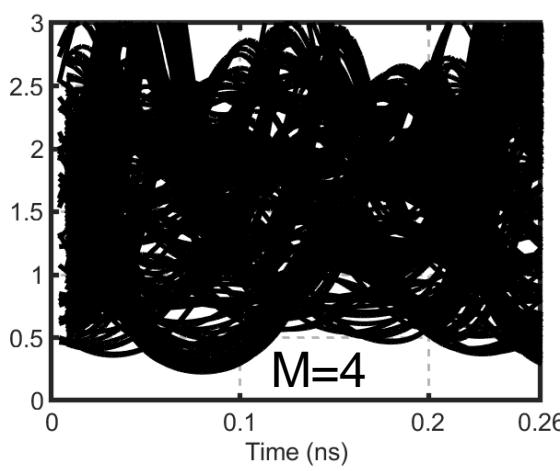

(e)

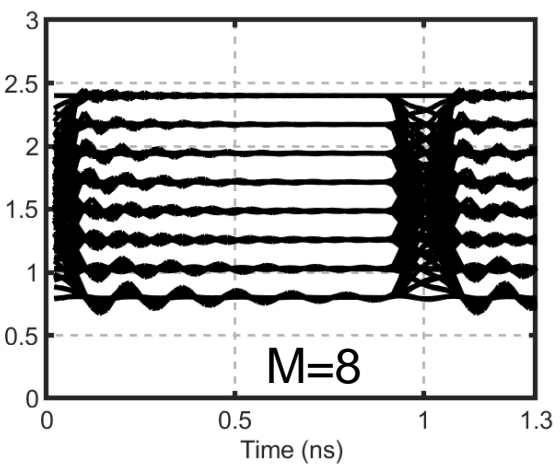

(c)

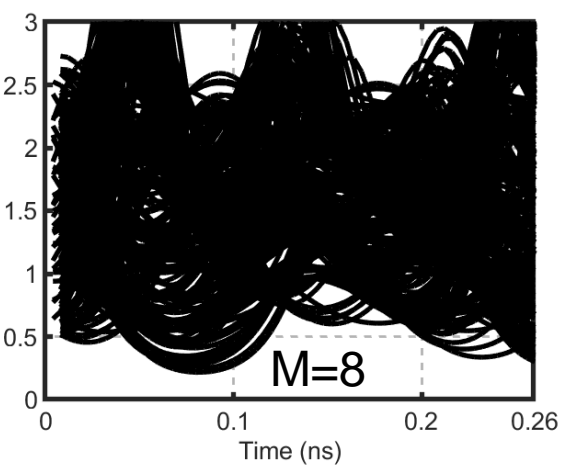

(f)

Figure 2. Eye patterns of the free-running lasers. (a) 2-level amplitude-shift keying (ASK) signal at 1-Gbaud data modulation. (b) 4-level ASK signal at 1-Gbaud data modulation. (c) 8-level ASK signal at 1-Gbaud data modulation. (d) 2-level ASK signal at 5-Gbaud data modulation. (e) 4-level ASK signal at 5-Gbaud data modulation. (f) 8-level ASK signal at 5-Gbaud data modulation.

Figure 3 shows the eye patterns of the OIL semiconductor lasers modulated by M-level ASK signals. It shows the output photon numbers for various M-levels and symbol-rates. Figure $3 \mathrm{a}-\mathrm{c}$ show the eye patterns of 2-, 4-, and 8-level ASK modulated signals at a 1Gbaud data, respectively. Figure 3d-f show the eye patterns of the 2-, 4-, and 8-level ASK modulated signals at a 5-Gbaud data, respectively.

Although the higher symbol-rate and the increased number of M-levels degrades the eye-opening performance, the degradation is not as drastic as that of the free-running laser. The eye openings of the OIL semiconductor lasers are much larger than those of the free-running laser. The eye patterns produce a decent modulation signal with reduced modulation-induced noise as compared to the free-running laser. It was found that the OIL lasers can significantly improve the M-level modulation performances for ultra-highcapacity systems owing to the enhanced laser modulation dynamics, such as increase in resonance frequency, reduction in turn-on-delay, and reduction in damping.

Figure $3 e, f$ show an asymmetry in the rising and falling shapes. The falling time of the photon number due to the temporal decrease of the modulation current is greater than the rising time of the photon number due to the temporal increase of the modulation current. The external photon injection to the OIL laser cavity maintains the level of the photon reservoir so that the time required to decrease the photon number in the cavity reservoir is longer than the case of rising signal. 


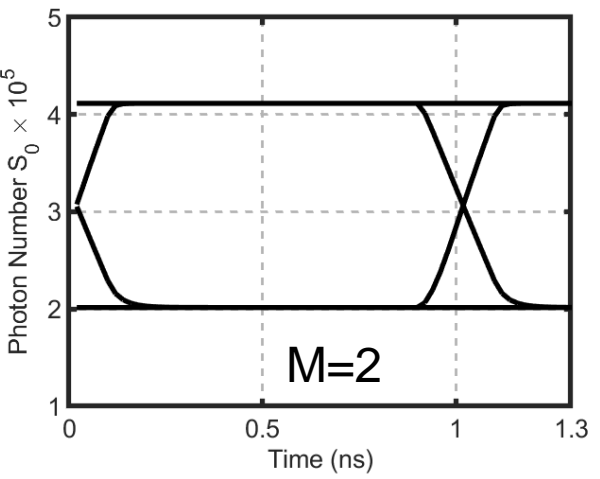

(a)

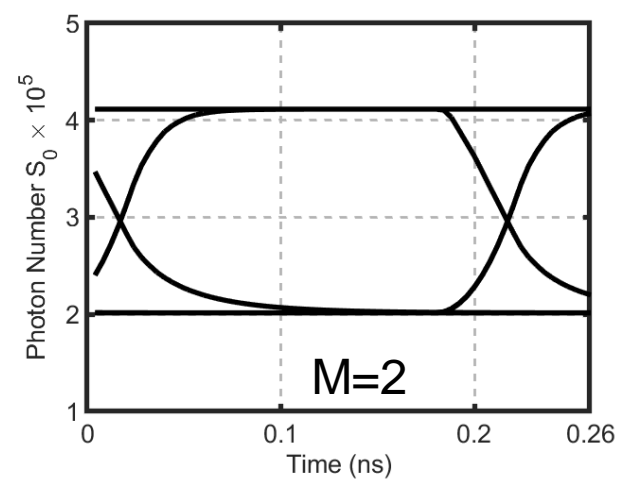

(d)

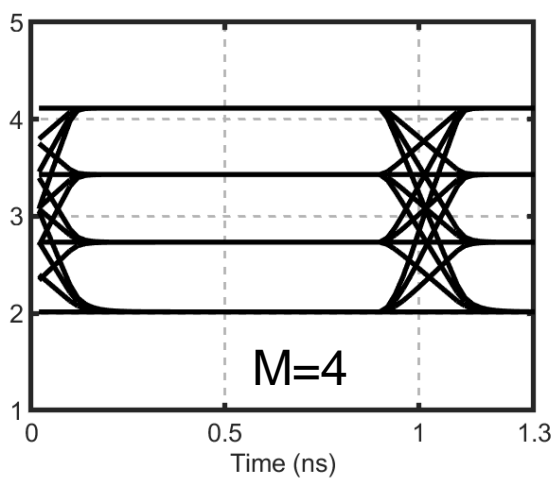

(b)

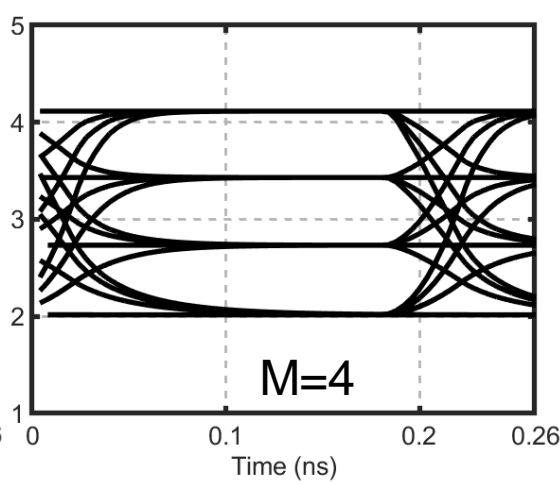

(e)

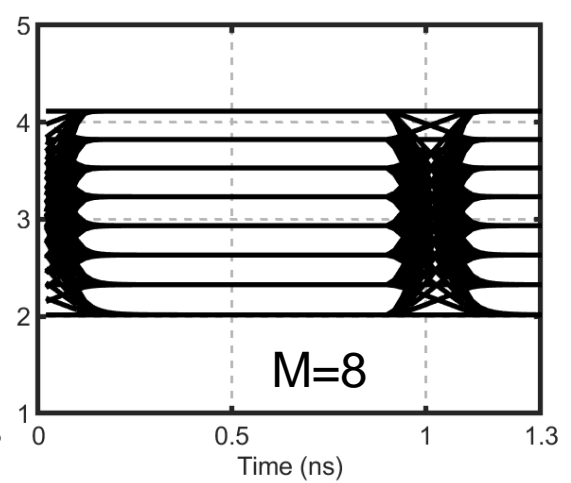

(c)

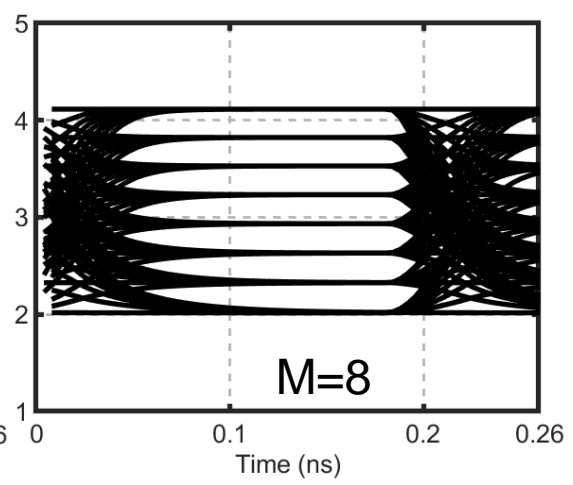

(f)

Figure 3. Eye pattern of the OIL semiconductor lasers. (a) 2-level amplitude-shift keying (ASK) signal at 1-Gbaud data modulation. (b) 4-level ASK signal at 1-Gbaud data modulation. (c) 8-level ASK signal at 1-Gbaud data modulation. (d) 2-level ASK signal at 5-Gbaud data modulation. (e) 4-level ASK signal at 5-Gbaud data modulation. (f) 8-level ASK signal at 5-Gbaud data modulation.

We calculated the Q-factor to evaluate the optical M-level modulation performance. The Q-factor plays an important role in quantifying the performance of a digital communication system. It is shown in Equation (6) [28]:

$$
Q_{k}=\frac{u_{k}-u_{k-1}}{\sigma_{k}+\sigma_{k-1}} \cdot(k=1,2,3, \cdots, \mathrm{M}-1)
$$

where $u_{k}$ and $u_{k-1}$ are the average values of the two adjacent signal levels in M-level ASK signals. The difference of $u_{k}$ and $u_{k-1}$ is divided by the sum of the standard deviations $\sigma_{k}$ and $\sigma_{k-1}$ for each signal level. For M-level ASK modulation, we calculated the Q-factors for the adjacent signals by the Equation (6) and divided the sum of the adjacent Q-factors by $(\mathrm{M}-1)$ to obtain the average $\mathrm{Q}$-factor.

Figure 4 shows the averaged Q-factors of the free-running and OIL semiconductor lasers as a function of various M-levels for 10-Gbaud and 20-Gbaud modulation. As the level of M-value in the M-level ASK modulation increases, the Q-factor decreases in both the free-running and OIL semiconductor lasers. The Q-factor also decreases as the data rate increases. The performance of $\mathrm{M}$-level modulation in terms of $\mathrm{Q}$-factor improves significantly for the OIL laser as compared to the free-running laser. It shows more than two times the improvement for the OIL laser $(\sim 0.7)$ as compared to the free-running laser $(\sim 0.3)$ at 10-Gbaud data rate with 5-level modulation. 


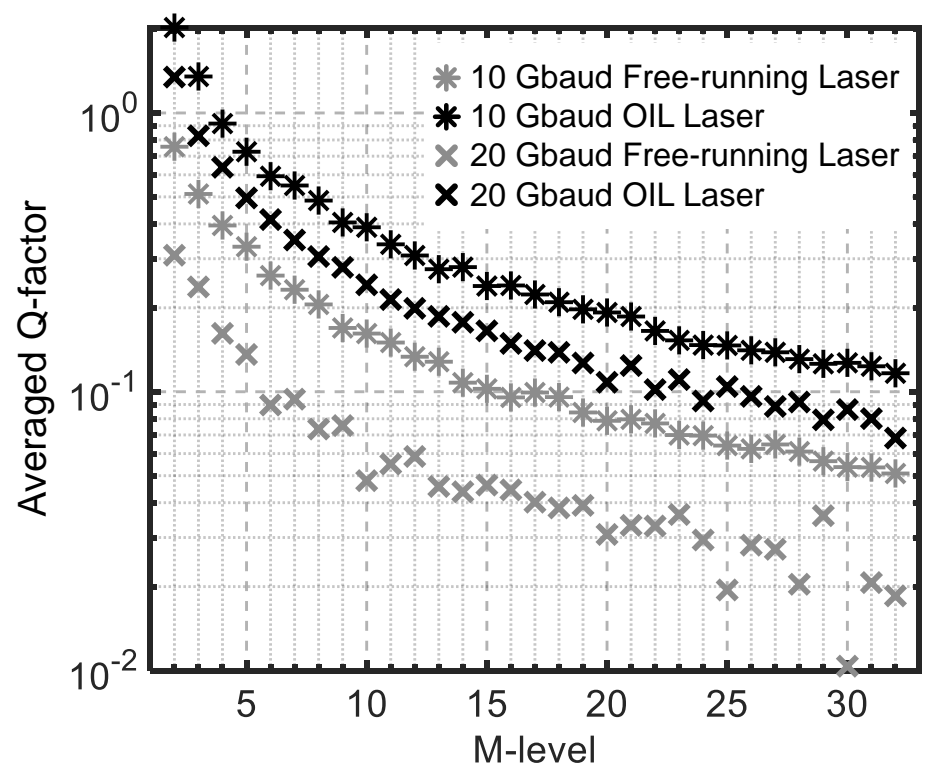

Figure 4. Averaged Q-factors of the free-running and the OIL semiconductor lasers as a function of various M-levels for 10-Gbaud and 20-Gbaud modulation. Q-factor: quality-factor; M-level: multi-level; OIL: optically injection locked.

Figure 5 shows the averaged Q-factors of the free-running and OIL semiconductor lasers as a function of various data rates for 2- and 4-level ASK signals. The Q-factor performance is maintained as high as 0.3 for the OIL laser with 2- and 4-level modulation cases for 50-Gbaud data rates, while it is significantly reduced to a low value for the freerunning laser. This clearly shows the potential of the M-level optical modulation based on the OIL laser for ultra-high-capacity data systems.

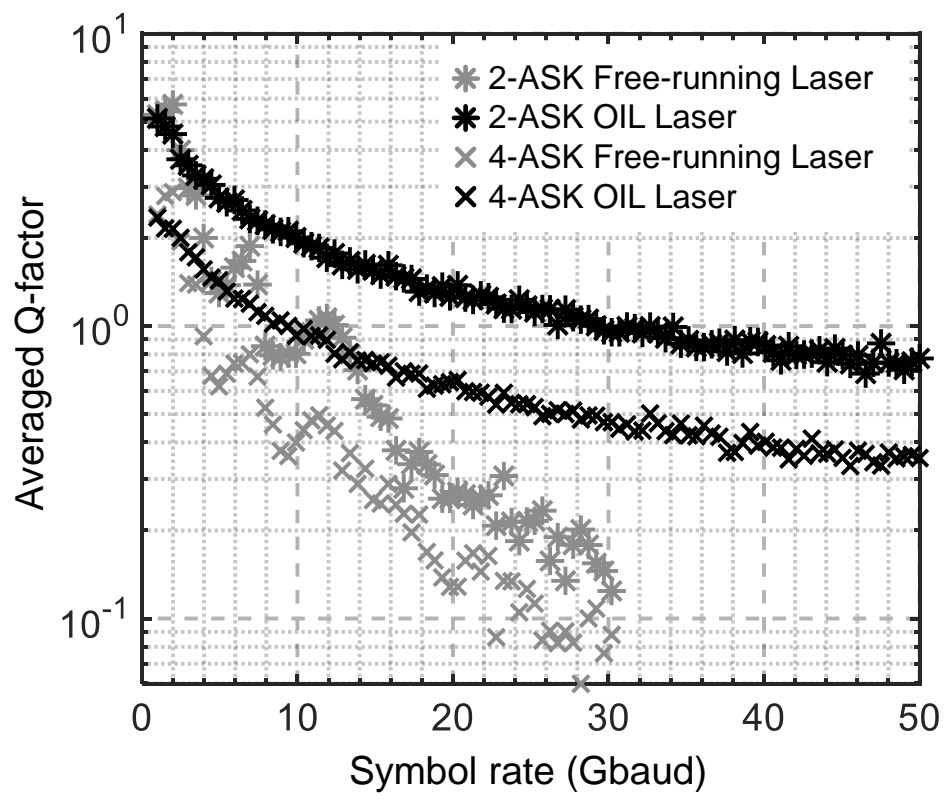

Figure 5. Averaged Q-factors of the free-running and the OIL semiconductor lasers as a function of various data rates for 2 and 4-level ASK signals. Q-factor: quality-factor; ASK: amplitude-shift keying; OIL: optically injection-locked.

We evaluated the dependence of Q-factor performance on the detuning frequency and injection ratio. Figure 6 shows the averaged Q-factors represented as gray-colored range in the locking map for two-level ASK signals at 10-Gbaud data modulation. The 
Q-factor exhibits an enhanced performance when the injection-locking parameters are adjusted in the region of negative detuning frequency and high injection ratio. The reason for this is because of the increased damping of the resonance of the OIL laser in the negative detuning range.

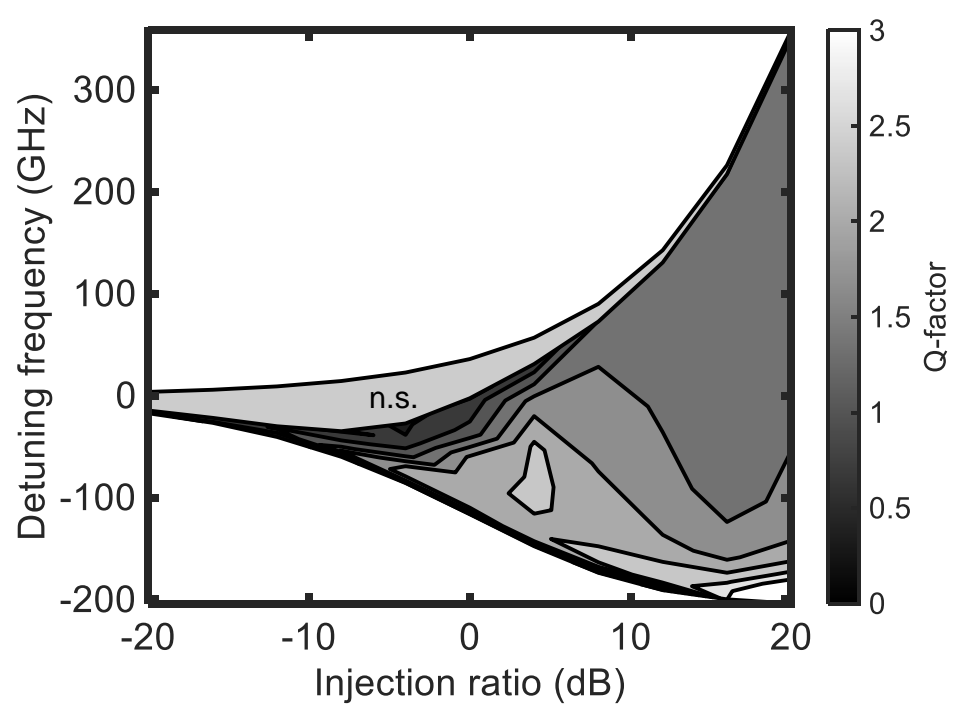

Figure 6. Q-factor dependence on the injection-locking parameters for two-level ASK signals at $10-G b a u d$ data modulation. The locking range is represented as a gray-scaled range that shows the Q-factor value. Q-factor: quality-factor; n.s.: unstable locking range.

\section{Discussion}

Direct modulation performances of optically injection-locked (OIL) lasers exhibit the significant improvement compared with a free-running laser [9-19]. Based on the theory of the modulation property improvement, we apply the multi-level (M-level) modulation signal to the OIL laser to increase data transmission capacity. We achieved the enhanced M-level modulation performance owing to the modulation bandwidth increase, chirp reduction, and turn-on delay reduction of the OIL laser. Furthermore, we found that the M-level modulation performance can be significantly improved when the OIL laser operates within the negative frequency detuning regime. This is due to the strong damping of the modulation dynamics of the OIL laser. The improved M-level optical modulation performance of the OIL laser can be applied to a wide range of various high-capacity optical systems, including optical wired/wireless communication, data centers, and photonic circuits. As a future work, we will confirm the simulation results by the experimental demonstrations.

\section{Conclusions}

We proposed a simple and efficient multi-level (M-level) optical modulation based on the direct modulation of optically injection-locked (OIL) lasers. We theoretically confirmed the potential of the OIL laser for ultra-high-capacity data systems by evaluating the eyediagram and Q-factor performances. The directly modulated OIL laser exhibited a wider eye-opening and a higher $\mathrm{Q}$-factor for a large value of the M-level modulation as compared to a free-running laser. They also exhibited high optical modulation performances for large data rates up to $50 \mathrm{Gbaud}$. We also found that the Q-factor performance is improved when the injection-locking parameters are adjusted in the region of negative detuning frequency and strong injection ratio. 
Author Contributions: All authors contributed considerably to this article. The article was conceived and structured by all authors. H.-K.S. and J.-H.C. conceived the simulation, analyzed the data, and wrote the paper. H.-S.J. performed the simulations and analyzed the data. All authors have read and agreed to the published version of the manuscript.

Funding: This research was funded by the National Research Foundation of Korea (NRF) under the Basic Science Research Program (NRF-2019R1I1A1A01058848, and NRF-2019R1F1A1040959).

Conflicts of Interest: The authors declare no conflict of interest. The funders had no role in the design of the study; in the collection, analyses, or interpretation of data; in the writing of the manuscript, or in the decision to publish the results.

\section{References}

1. Winzer, P.J.; Essiambre, R.J. Advanced Optical Modulation Formats. Proc. IEEE 2006, 94, 952-985. [CrossRef]

2. Karinou, F.; Borkowski, R.; Zibar, D.; Roudas, I.; Vlachos, K.G.; Monroy, I.T. Advanced Modulation Techniques for HighPerformance Computing Optical Interconnects. IEEE J. Sel. Top. Quantum Electron. 2013, 19, 3700614. [CrossRef]

3. Olmedo, M.I.; Zuo, T.; Jensen, J.B.; Zhong, Q.; Xu, X.; Popov, S.; Monroy, I.T. Multiband Carrierless Amplitude Phase Modulation for High Capacity Optical Data Links. J. Lightwave Technol. 2014, 32, 798-804. [CrossRef]

4. Dong, P.; Liu, X.; Chandrasekhar, S.; Buhl, L.L.; Aroca, R.; Chen, Y. Monolithic Silicon Photonic Integrated Circuits for Compact $100+\mathrm{Gb} / \mathrm{s}$ Coherent Optical Receivers and Transmitters. IEEE J. Sel. Top. Quantum Electron. 2014, 20, 150-157. [CrossRef]

5. Cheng, Q.; Bahadori, M.; Glick, M.; Rumley, S.; Bergman, K. Recent advances in optical technologies for data centers: A review. Optica 2018, 5, 1354-1370. [CrossRef]

6. Lau, K.S.; Wong, K.H.; Chan, T.L.; Yeung, S.K. An Economical Piezoelectric Phase Modulator for Fiber Optic Sensors. Appl. Opt. 1996, 35, 6836-6838. [CrossRef] [PubMed]

7. Saleh, B.E.A.; Teich, M.C. Fundamentals of Photonics, 2nd ed.; John Wiley \& Sons, Inc.: New York, NY, USA, $1991 ;$ pp. 819-856.

8. Liu, Z.; Kakande, J.; Kelly, B.; O'Carroll, J.; Phelan, R.; Richardson, D.J.; Slavík, R. Modulator-free quadrature amplitude modulation signal synthesis. Nat. Commun. 2014, 5, 5911. [CrossRef]

9. Sung, H.K.; Lau, E.K.; Wu, M.C. Optical Properties and Modulation Characteristics of Ultra-Strong Injection-Locked Distributed Feedback Lasers. IEEE J. Sel. Top. Quantum Electron. 2007, 13, 1215-1221. [CrossRef]

10. Simpson, T.B.; Liu, J.M.; Huang, K.F.; Tai, K.; Clayton, C.M.; Gavrielides, A.; Kovanis, V. Cavity enhancement of resonant frequencies in semiconductor lasers subject to optical injection. Phys. Rev. A 1995, 52, R4348-R4351. [CrossRef] [PubMed]

11. Lee, H.L.T.; Ram, R.J.; Kjebon, O.; Schatz, R. Bandwidth enhancement and chirp reduction in DBR lasers by strong optical injection. In Proceedings of the Conference on Lasers and Electro-Optics (CLEO 2000), San Francisco, CA, USA, 7-12 May 2000

12. Murakami, A.; Kawashima, K.; Atsuki, K. Cavity resonance shift and bandwidth enhancement in semiconductor lasers with strong light injection. IEEE J. Quantum Electron. 2003, 39, 1196-1204. [CrossRef]

13. Chrostowski, L.; Zhao, X.; Chang-Hasnain, C.J.; Shau, R.; Ortsiefer, M.; Amann, M. 50 GHz Directly-Modulated Injection-Locked $1.55 \mu \mathrm{m}$ VCSELs. In Proceedings of the Optical Fiber Communication Conference, Anaheim, CA, USA, 6 March 2005.

14. Iwashita, K.; Nakagawa, K. Suppression of mode partition noise by laser diode light injection. IEEE J. Quantum Electron. 1985, 18, 1669-1674. [CrossRef]

15. Lin, C.; Mengel, F. Reduction of frequency chirping and dynamic linewidth in high-speed directly modulated semiconductor lasers by injection locking. Electron. Lett. 1984, 20, 1073-1075. [CrossRef]

16. Olsson, N.; Temkin, H.; Logan, R.; Johnson, L.; Dolan, G.; van der Ziel, J.; Campbell, J. Chirp-free transmission over 82.5 km of single mode fibers at $2 \mathrm{Gbit} / \mathrm{s}$ with injection locked DFB semiconductor lasers. J. Lightwave Technol. 1985, 3, 63-67. [CrossRef]

17. Mohrdiek, S.; Burkhard, H.; Walter, H. Chirp reduction of directly modulated semiconductor lasers at $10 \mathrm{~Gb} / \mathrm{s}$ by strong CW light injection. J. Lightwave Technol. 1994, 12, 418-424. [CrossRef]

18. Meng, X.J.; Chau, T.; Wu, M.C. Improved intrinsic dynamic distortions in directly modulated semiconductor lasers by optical injection locking. IEEE Trans. Microw. Theory Tech. 1999, 47, 1172-1176. [CrossRef]

19. Meng, X.J.; Chau, T.; Tong, D.T.K.; Wu, M.C. Suppression of second harmonic distortion in directly modulated distributed feedback lasers by external light injection. Electron. Lett. 1998, 34, 2040-2041. [CrossRef]

20. Yamaoka, S.; Diamantopoulos, N.-P.; Nishi, H.; Nakao, R.; Fujii, T.; Takeda, K.; Hiraki, T.; Tsurugaya, T.; Kanazawa, S.; Tanobe, H.; et al. Directly modulated membrane lasers with $108 \mathrm{GHz}$ bandwidth on a high-thermal-conductivity silicon carbide substrate. Nat. Photon. 2021, 15, 28-35. [CrossRef]

21. Matsui, Y.; Schatz, R.; Che, D.; Khan, F.; Kwakernaak, M.; Tsurugi, S. Low-chirp isolator-free 65-GHz-bandwidth directly modulated lasers. Nat. Photonics 2021, 15, 59-63. [CrossRef]

22. Kobayashi, W.; Ito, T.; Yamanaka, T.; Fujisawa, T.; Shibata, Y.; Kurosaki, T.; Kohtoku, M.; Tadokoro, T.; Sanjoh, H. 50-Gb/s Direct Modulation of a 1.3- $\mu \mathrm{m}$ InGaAlAs-Based DFB Laser With a Ridge Waveguide Structure. IEEE J. Sel. Top. Quantum Electron. 2013, 19, 1500908. [CrossRef]

23. Nguyen, A.H.; Cho, J.H.; Bae, H.J.; Sung, H.K. Side-lobe Level Reduction of an Optical Phased Array Using Amplitude and Phase Modulation of Array Elements Based on Optically Injection-Locked Semiconductor Lasers. Photonics 2020, 7, 20. [CrossRef] 
24. Cho, J.H.; Cho, C.H.; Sung, H.K. Theoretical performance evaluation of optical complex signals based on optically injection-locked semiconductor lasers. IEEE J. Sel. Top. Quantum Electron. 2019, 25, 1-9. [CrossRef]

25. Lau, E.K.; Wong, L.J.; Wu, M.C. Enhanced Modulation Characteristics of Optical Injection-Locked Lasers: A Tutorial. IEEE J. Sel. Top. Quantum Electron. 2009, 15, 618-633. [CrossRef]

26. Lee, H.; Cho, J.H.; Sung, H.K. Enhancement of the phase-modulation range by using cascaded injection-locked semiconductor lasers. J. Korean Phys. Soc. 2016, 68, 756-761. [CrossRef]

27. Lee, H.; Cho, J.H.; Sung, H.K. Theoretical analysis of a method for extracting the phase of a phase-amplitude modulated signal generated by a direct-modulated optical injection-locked semiconductor laser. Opt. Eng. 2017, 56, 056112. [CrossRef]

28. Abdullah, M.F.L.; Talib, R. Multilevel signal analyzer tool for optical communication system. Int. J. Electr. Comput. Eng. 2012, 2, 529. [CrossRef] 